\title{
Development of HER2-targeted Therapies for Gastrointestinal Cancer
}

\author{
Kentaro Sawada, Yoshiaki Nakamura and Kohei Shitara \\ Department of Gastroenterology and Gastrointestinal Oncology, National Cancer Centre Hospital East, Chiba, Japan
}

DOI: https://doi.org/10.17925/EOH.2020.16.1.29

$\mathrm{H}$

uman epidermal growth factor receptor 2 (HER2) amplification is an important molecular mechanism underlying carcinogenesis and is associated with various types of cancer. Although the advancement of HER2-targeted therapy has been the most pronounced in breast cancer, interest has emerged in exploring the efficacy of HER2-targeted therapies in gastrointestinal (GI) cancers. In particular, the addition of trastuzumab to first-line chemotherapy has improved the overall survival of patients with HER2-positive gastric or oesophagogastric junction cancer. Although subsequent trials involving lapatinib, ado-trastuzumab emtansine (T-DM1), and pertuzumab have failed to show significant survival benefits for HER2-positive gastric or oesophagogastric junction cancer, several trials are currently ongoing. HER2-targeted therapy has also been tested in patients with other GI cancers. Some combination therapies, such as trastuzumab plus pertuzumab, have shown promising results in single-arm phase II studies. Moreover, trials of novel anti-HER2 agents, including trastuzumab deruxtecan (T-DXd), tucatinib and margetuximab - which demonstrated improvement of clinical outcomes in breast cancer - are ongoing for GI cancers. In this review, we provide an overview of the current status of HER2-targeted therapies and focus on future perspectives for overcoming issues in the treatment of HER2-positive GI cancer.

\section{Keywords}

Gastrointestinal cancer, HER2, trastuzumab,

T-DXd, targeted therapy, gastric

Disclosure: Yoshiaki Nakamura reports the research funding from Taiho Pharmaceutical. Kohei Shitara reports paid consulting or advisory roles for Astellas, Lilly, Bristol-Myers Squibb, Takeda, Pfizer, Ono and MSD;

honoraria from Novartis, AbbVie, and Yakult; and research funding from Astellas, Lilly, Ono, Sumitomo Dainippon, Daiichi Sankyo, Taiho, Chugai, MSD and Medi Science. Kentaro Sawada has no financial or non-financial relationships or activities to declare in relation to this article.

Review Process: Double-blind peer review.

Compliance with Ethics: This article involves a review of the literature and did not involve any studies with human or animal subjects performed by any of the authors.

Authorship: The named authors meet the International Committee of Medical Journal Editors (ICMJE) criteria for authorship of this manuscript, take responsibility for the integrity of the work as a whole, and have given final approval for the version to be published. Access: This article is freely accessible at touchONCOLOGY.com (c) Touch Medical Media 2020.

Received: 4 March 2020

Accepted: 19 May 2020

Published Online: 31 July 2020

Citation: European Oncology \& Haematology. 2020;16(1):29-38

Corresponding Author: Kohei Shitara, Department of Gastroenterology and Gastrointestina Oncology, National Cancer Center Hospital East

6-5-1 Kashiwanoha, Kashiwa, Chiba 277-8577, Japan. E:kshitara@east.ncc.go.jp

Support: No funding was received in

the publication of this article.
Human epidermal growth factor receptor 2 (HER2) is encoded by a proto-oncogene with important roles in the promotion of cell proliferation, differentiation and angiogenesis via the activation of Phosphatidylinositol-3-kinase (PI3K)/protein kinase B (AKT)/mammalian target of rapamycin (mTOR) and mitogen-activated protein kinase (MAPK) downstream signalling after it forms homodimers or heterodimers with other HER family members. ${ }^{1-2}$ HER2 overexpression, mainly caused by gene amplification, allows HER2 activation, even in the absence of ligand binding. ${ }^{3}$

HER2 amplification is the most frequently observed in breast cancer; accordingly, the status of HER2-targeted therapy is the most advanced in this cancer type. Although HER2 overexpression is a poor prognostic factor in breast cancer, HER2-targeted therapy has improved clinical outcomes dramatically. ${ }^{4-5}$ A variety of agents - including trastuzumab, pertuzumab, lapatinib, ado-trastuzumab emtansine (T-DM1) and neratinib - have been approved for HER2-positive breast cancer. Recently, novel anti-HER2 agents - including trastuzumab deruxtecan (T-DXd), tucatinib and margetuximab - have demonstrated significant improvements in clinical outcome. $^{6-8}$ T-DXd is an antibody-drug conjugate composed of an anti-HER2 antibody, a cleavable tetrapeptide-based linker, and a cytotoxic topoisomerase I inhibitor. ${ }^{9}$ T-DXd has shown a remarkable efficacy in DESTINY-Breast01 (A study of DS-8201a in metastatic breast cancer previously treated with trastuzumab emtansine [T-DM1]; ClinicalTrials.gov identifier: NCT03248492), a multicentre, single-arm, phase II study including 184 patients with HER2-positive breast cancer who previously received HER2-targeted therapies, leading to US Food and Drug Administration approval. ${ }^{6}$

Recent genotyping studies have revealed HER2 amplification in multiple cancer types. In light of the successes with trastuzumab and other agents in with HER2-positive breast cancer, interest has emerged in exploring the efficacy of HER2-targeted therapies for other cancer types. Specifically, gastrointestinal (GI) cancer is the second-most common cancer with HER2 amplification. Multiple HER2-targeted therapies have been developed and are in clinical trials. In this review, we focus on recent developments in HER2-targeted therapies and future perspectives for using them to treat GI cancers, including gastric cancer, colorectal cancer, oesophageal cancer, biliary tract cancer and pancreatic cancer.

\section{Gastroesophageal junction cancer}

Among Gl cancers, the greatest advances in HER2-targeted therapy have been achieved for gastroesophageal junction (GEJ) cancers. The frequency of HER2 amplification in gastric cancers is $15-25 \%$ and is higher than the overall frequency in GI cancer. ${ }^{10-13}$ In particular, GEJ cancers have a higher rate of HER2 amplification (32.2\%) than those of gastric cancers (21.4\%); and intestinal tumours also have a higher rate of HER2 amplification (31.8\%) than those with a diffuse type (6.1\%). ${ }^{13}$ 
Table 1: The efficacy of HER2-targeted therapies in gastric cancer

\begin{tabular}{|c|c|c|c|c|c|c|c|c|}
\hline Trial & $\mathrm{N}$ & Phase & Line & Definition of HER2-positive & Treatment & OS (months) & PFS (months) & ORR (\%) \\
\hline $\begin{array}{l}\text { ToGA }^{16,18} \\
\text { (NCT01041404) }\end{array}$ & 594 & III & First & IHC 3+ and/or ISH-positive & $\begin{array}{l}\text { Capecitabine or } 5 \text {-FU, } \\
\text { cisplatin } \pm \text { trastuzumab }\end{array}$ & 13.8 versus 11.1 & 6.7 versus 5.5 & 47.3 versus 4.5 \\
\hline $\begin{array}{l}\text { T-ACT } \\
\text { (WJOG7112G) }\end{array}$ & 99 & $\|$ & Second & $\begin{array}{l}\text { IHC } 3+\text { or IHC } 2+\text { and } \\
\text { FISH-positive }\end{array}$ & $\begin{array}{l}\text { Paclitaxel } \pm \text { trastuzumab } \\
\text { (beyond progression) }\end{array}$ & 10.0 versus 10.2 & 3.2 versus 3.7 & 31.6 versus 33.3 \\
\hline $\begin{array}{l}\text { LOGiC }^{20,21} \\
\text { (NCT00680901) }\end{array}$ & 545 & III & First & IHC 3+ and/or ISH-positive & $\begin{array}{l}\text { Capecitabine, oxaliplatin } \\
\pm \text { lapatinib }\end{array}$ & 12.5 versus 10.5 & 6.0 versus 5.4 & 53 versus 39 \\
\hline $\begin{array}{l}\text { TyTAN }^{22,23} \\
\text { (NCT00486954) }\end{array}$ & 261 & III & Second & ISH-positive & Paclitaxel \pm lapatinib & 11.0 versus 8.9 & 5.9 versus 4.4 & 27 versus 9 \\
\hline $\begin{array}{l}\text { GATSBY24,25 } \\
\text { (NCT01641939) }\end{array}$ & 302 & III & Second & $\begin{array}{l}\text { IHC 3+ or IHC 2+ and } \\
\text { FISH-positive }\end{array}$ & $\begin{array}{l}\text { T-DM1 versus paclitaxel/ } \\
\text { docetaxel }\end{array}$ & 7.9 versus 8.6 & 2.7 versus 2.9 & 20.6 versus 19.6 \\
\hline $\begin{array}{l}\text { JACOB }^{26,27} \\
\text { (NCT01774786) }\end{array}$ & 780 & III & First & $\begin{array}{l}\text { IHC } 3+\text { or IHC } 2+\text { and } \\
\text { FISH-positive }\end{array}$ & $\begin{array}{l}\text { Capecitabine or 5-FU, } \\
\text { cisplatin, trastuzumab } \pm \\
\text { pertuzumab }\end{array}$ & 17.5 versus 14.2 & 8.5 versus 7.0 & 58.7 versus 48.3 \\
\hline $\begin{array}{l}\text { DESTINY- } \\
\text { Gastric0128,29 } \\
\text { (NCT03329690) }\end{array}$ & 187 & $\|$ & $\begin{array}{l}\text { Third or } \\
\text { later }\end{array}$ & $\begin{array}{l}\mathrm{IHC} 3+\text { or } \mathrm{IHC} 2+\text { and } \\
\text { FISH-positive }\end{array}$ & $\begin{array}{l}\text { Trastuzumab deruxtecan } \\
\text { versus physician's choice of } \\
\text { chemotherapy }\end{array}$ & 12.5 versus 8.4 & 5.6 versus 3.5 & 51.0 versus 14.0 \\
\hline
\end{tabular}

5-FU = 5-fluorouracil; FISH = fluorescence in situ hybridisation; HER2 = human epidermal growth factor receptor 2; IHC = immunohistochemistry; ISH = in situ hybridisation; $O R R=$ objective response rate; $O S=$ overall survival; PFS = progression-free survival; T-DM1 = ado-trastuzumab emtansine.

A distinct characteristic of HER2-positive gastric cancer, including GEJ cancer, that differentiates it from HER2-positive breast cancer is intra-tumoural heterogeneity, ${ }^{14-15}$ which is characterised as the presence of areas with different HER2 immunohistochemistry (IHC) scores within the same tumour. Moreover, the criteria for HER2 positivity differ slightly between gastric cancer and breast cancer. In the ToGA trial, which was the first randomised phase III study of HER2-positive gastric or GEJ cancer, HER2 positivity was defined as either: (1) IHC 3+ (defined as moderate to strong, complete or basolateral membrane staining in $>10 \%$ of tumour cells); or (2) in situ hybridisation (ISH)-positive (defined as a HER2:CEP17 ratio of $>2) .{ }^{16}$ Based on these criteria, $22.1 \%$ of tumours were classified as HER2 positive. However, because the IHC $1+/ 0$ and the ISH-positive subgroup did not show a clinical benefit in this trial, HER2 positivity has since been redefined as $\mathrm{IHC} 3+$ or $\mathrm{IHC} 2+$ and ISH-positive tumours in clinical settings. ${ }^{17}$

In the ToGA trial (A study of herceptin [trastuzumab] in combination with chemotherapy compared with chemotherapy alone in patients with HER2-positive advanced gastric cancer; ClinicalTrials.gov identifier: NCT01041404), 584 patients with HER2-positive gastric or GEJ cancer were recruited according to the above criteria and were randomised to receive fluoropyrimidine and cisplatin with trastuzumab (monoclonal antibody targeting HER2) or placebo. ${ }^{16,18}$ In the trastuzumab group, median overall survival (OS) was 13.8 months and median progression-free survival (PFS) was 6.7 months. These durations were significantly longer than those in the placebo group (i.e., 11.1 and 5.5 months, respectively) (Table 1)..$^{16,18-29}$ In particular, in patients who were IHC 3+ or 2+ and fluorescence in situ hybridisation (FISH)-positive, trastuzumab with chemotherapy demonstrated remarkable efficacy, with a median OS of 16.0 months (hazard ratio [HR] 0.65, 95\% confidence interval [Cl] 0.51-0.83), whereas the survival benefit was not observed (HR 1.07, 95\% $\mathrm{Cl} 0.70-1.62)$ in patients with low levels of HER2 protein (IHC 0/1+ and FISH-positive). According to the survival benefit in patients with high HER2 expression, IHC 3+ or IHC 2+ and ISH-positive disease is considered positive for gastric cancer.

On the other hand, the efficacy of trastuzumab beyond disease progression was not confirmed in gastric cancer. In the
T-ACT (Randomized, phase II study of trastuzumab beyond progression in patients with HER2-positive advanced gastric or gastroesophageal junction cancer [WJOG7112G]) randomised phase II trial, 91 patients with HER2-positive gastric or GEJ cancer with disease progression after first-line chemotherapy plus trastuzumab, were assigned to groups treated with paclitaxel with or without trastuzumab. ${ }^{19}$ The median OS and PFS were 10.0 and 3.2 months in the trastuzumab combination group and 10.2 and 3.7 months in the paclitaxel monotherapy group (OS: HR 1.23, $p=0.20$; PFS 0.91, $p=0.33$ ), which was not significant (Table 1). On exploratory analyses, HER2 expression was lost after first-line chemotherapy in 11 of 16 (69\%) patients whose tumour tissues were available, which might cause no clinical benefit by trastuzumab beyond disease progression.

Lapatinib, a tyrosine kinase inhibitor (TKI) that binds to the intracellular tyrosine kinase domains of epidermal growth factor receptor (EGFR) and HER2, blocking autophosphorylation and downstream signalling, has been investigated for gastric cancer. In the TRIO-013/LOGiC trial (Lapatinib optimization study in ErbB2 [HER2] positive gastric cancer: a phase III global, blinded study designed to evaluate clinical endpoints and safety of chemotherapy plus lapatinib; ClinicalTrials.gov identifier: NCT00680901), 545 patients with gastric or GEJ cancer harbouring HER2 amplifications, defined as IHC 3+ or ISH-positive regardless of HER2 IHC score, were assigned to groups receiving capecitabine and oxaliplatin plus either lapatinib or placebo in the first-line setting. ${ }^{20,21}$ The primary endpoint, median OS, was 12.2 months in the lapatinib arm and 10.5 months in the placebo arm, which was not significant (HR, 0.91, $\mathrm{p}=0.349$ ) (Table 1). In a pre-planned exploratory subgroup analysis, OS benefit was suggested in patients under 60 years of age and those of Asian ethnicity receiving lapatinib. On the other hand, no statistically significant correlations were observed between HER2 IHC status and survival.

Tykerb with taxol in Asian HER2-positive gastric cancer (TyTan; ClinicalTrials.gov identifier: NCT00486954) is a phase III trial involving 261 patients with HER2 amplification-positive gastric or GEJ cancer regardless of HER2 IHC score, previously treated with trastuzumab. 22,23 Enrolled patients were randomised to receive paclitaxel with or without 
lapatinib as the second-line chemotherapy. The median OS was 11.0 months for lapatinib plus paclitaxel versus 8.9 months for paclitaxel alone with no significant difference $(p=0.104$ ) (Table 1). However, lapatinib is not approved for gastric cancer because neither the LOGiC nor the TyTan trials showed significant improvement in OS in the intent-totreat population. The lack of survival benefit in LoGiC and TyTan may be attributed to inclusion of patients with low HER2 expression, which was suggested not to have clinical efficacy by HER2-targeted therapy based on results of the ToGA study. Indeed, in a subgroup analysis of the TyTan trial, patients with IHC $3+(101 / 192,53 \%)$ in the lapatinib plus paclitaxel group had better OS (14.0 versus 7.6 months) and PFS (5.6 versus 4.2 months) than those in the paclitaxel-only group. The LoGiC trial also included 81 (17\%) patients with HER2 IHC 0/1+ disease, though this trial did not show significant OS improvement even in patients with $1 \mathrm{HC} 2+/ 3+$ disease. These results indicated that HER2-targeted therapy needs to be developed for gastric cancer with high HER2 expression.

T-DM1 is an antibody-drug conjugate composed of trastuzumab, a thioether linker, and the potent microtubule inhibitor, DM1. In the GATSBY study (A study of trastuzumab emtansine versus taxane in participants with human epidermal growth factor receptor 2 (HER2)-positive advanced gastric cancer; ClinicalTrials.gov identifier: NCT01641939), an open-label, adaptive phase II/III trial, the efficacy of T-DM1 was evaluated in comparison with taxane for patients with previously treated HER2-positive metastatic gastric or GEJ cancer. ${ }^{24,25}$ In this study, patients who were IHC $3+$ or $2+$ and FISH-positive were eligible. Seventy-seven per cent of enrolled patients had received HER2-targeted therapy as first-line chemotherapy (almost all containing trastuzumab), and were randomly assigned to receive either T-DM1 (2.4 mg/kg weekly) or taxane (2:1). OS was not improved in the T-DM1 group compared with the taxane group (median 7.9 versus 8.6 months). Furthermore, median PFS was also not improved (2.7 versus 2.9 months) (Table 1). Although T-DM1 has previously shown activity in patients with HER2-positive metastatic breast cancer who had progressed during or after HER2-targeted therapy, GATSBY could not show clinical benefit in HER2-postive gastric cancer compared with standard chemotherapy. ${ }^{30}$ One possible reason for this discrepancy is that emtansine might be less active in gastric cancer; vinca alkaloids, which inhibit microtubule polymerisation, seem to be less active in gastric cancer than in other cancers. Another possible reason is alteration of HER2 status after first-line chemotherapy with trastuzumab. Although most patients were selected on the basis of archival HER2 status in this study, there is evidence that HER2 status might be altered during progression on trastuzumab because of changes in the molecular profile of the tumour. ${ }^{3}$ In addition, HER2 overexpression has a higher incidence of intra-tumoural heterogeneity in gastric cancer compared with breast cancer. The focality of HER2 overexpression might affect the activity of T-DM1 because it only targets HER2-overexpressed cancer cells without bystander effect for surrounding HER2-negative cells due to non-cleavable linker.

Pertuzumab is a humanised monoclonal antibody, which binds a different epitope than the one bound by trastuzumab, that inhibits HER2 heterodimerisation. The CLEOPATRA study (A study to evaluate pertuzumab + trastuzumab + docetaxel vs. placebo + trastuzumab + docetaxel in previously untreated HER2-positive metastatic breast cancer; ClinicalTrials.gov identifier: NCT00567190) has demonstrated that the addition of pertuzumab to trastuzumab plus docetaxel for HER2-positive breast cancer can improve clinical outcomes. ${ }^{32,33}$ In gastric cancer, the efficacy of pertuzumab was investigated in the JACOB study (A study of pertuzumab in combination with trastuzumab and chemotherapy in participants with human epidermal growth factor receptor 2
(HER2)-positive metastatic gastroesophageal junction or gastric cancer; ClinicalTrials.gov identifier: NCT01774786), a randomised phase III trial to evaluate the efficacy of the addition of pertuzumab to trastuzumab plus capecitabine or 5-fluorouracil and cisplatin for patients with metastatic HER2-positive gastric or GEJ cancer. ${ }^{26,27}$ In this study, HER2 positivity criteria was same as that of GATSBY trial (IHC $3+$ or 2+ and FISH-positive). The median PFS was longer in the pertuzumab group than in the control group ( 8.5 versus 7.0 months; $p=0.0001$ ), and objective response rate (ORR) also improved ( 56.7 versus $48.3 \% ; p=0.026$ ). The primary endpoint, OS, in the pertuzumab group tended to be better than that of the experimental group (17.5 versus 14.2 months), but the difference was not statistically significant (HR $0.84, p=0.057$ ) (Table 1). It was difficult to assess which patients might be more likely to benefit from pertuzumab, because the HR for OS was consistent across patient subgroups. The relative dose intensity of chemotherapy treatment was numerically reduced in the pertuzumab group compared with the placebo group because of increasing of grade 3 or worse adverse events such as diarrhoea. This might also have played a part in the study outcome.

T-DXd, as described above, is an antibody-drug conjugate agent recently approved for breast cancer. Among patients in a phase Ib study, those with HER2-positive gastric or GEJ cancer post-trastuzumab were assigned in part IIb. Tumour HER2 status was assessed using archival samples. A total of 44 patients with HER2-positive gastric or GEJ cancer received 5.4 or $6.4 \mathrm{mg} / \mathrm{kg}$ T-DXd. Finally, 19 of 44 patients (43.2\%) achieved an objective response, with 35 of 44 (79.5\%) achieving disease control. After a median follow-up of 5.5 months, the median OS and PFS were 12.8 and 5.6 months, respectively (Table 1). ${ }^{28}$ Based on these promising results, the phase ॥ DESTINY-Gastric01 trial (DS-8201a in human epidermal growth factor receptor 2 (HER2)-expressing gastric cancer; ClinicalTrials.gov identifier: NCT03329690), for patients with previously treated HER2-positive (IHC 3+ or 2+ and FISH-positive) metastatic gastric or GEJ cancer, was performed. ${ }^{28,29}$ The results were reported recently; OS was longer with T-DXd than with the physician's choice of chemotherapy (median, 12.5 versus 8.4 months; $\mathrm{p}=0.01$ ) and ORR in T-DXd was higher than that of chemotherapy (51 versus $14 \%, p<0.001) .{ }^{28}$ Accordingly, the approval of this drug for clinical use is anticipated in Japan, where the SAKIGAKE designation system to promote driving early practical application for innovative pharmaceutical products has been granted for this indication.

\section{Colorectal cancer}

The incidence of HER2 amplification is $1-5 \%$ in unselected colorectal cancer (CRC). ${ }^{34-37}$ HER2 amplification is more frequent in CRC harbouring wild-type RAS/BRAF (5-8\%) than in CRC harbouring mutant RAS/BRAF. HER2 is not only an oncogenic driver, but also is associated with poor efficacy of anti-EGFR antibody treatment, based on preclinical and clinical data..$^{38-40}$

A preclinical, multi-armed, Italian study, using large xenograft cohorts from 85 patient-derived, genetically characterised metastatic CRC (mCRC) samples ('xenopatients') revealed that combining the anti-HER2 agents trastuzumab and lapatinib induced overt, long-lasting tumour regression in HER2-amplified xenopatients, although each drug alone was not effective. ${ }^{40}$ Based on these data, the efficacy of dual HER2-targeted therapy was evaluated in the HERACLES trial (Evaluation of trastuzumab in combination with lapatinib or pertuzumab in combination with trastuzumab-emtansine to treat patients with HER2-positive metastatic colorectal cancer; ClinicalTrials.gov identifier: NCT03225937), a multicentre, open-label phase II trial for patients with HER2-positive MCRC and wild-type KRAS exon 2 after standard 
Table 2: The efficacy of HER2-targeted therapies in colorectal cancer

\begin{tabular}{|c|c|c|c|c|c|c|c|c|}
\hline Trial & $\mathrm{N}$ & Phase & Line & Definition of HER2-positive & Treatment & $\begin{array}{l}\text { OS } \\
\text { (months) }\end{array}$ & $\begin{array}{l}\text { PFS } \\
\text { (months) }\end{array}$ & ORR (\%) \\
\hline $\begin{array}{l}\text { HERACLES } 35,41 \\
\text { (NCT03225937) }\end{array}$ & 27 & $\|$ & Refractory & $\begin{array}{l}\text { IHC } 3+\text { or IHC } 2+\text { and } \\
\text { FISH-positive }\end{array}$ & Trastuzumab + lapatinib & 11.5 & 5.2 & 30 \\
\hline $\begin{array}{l}\text { MyPathway }{ }^{42,43} \\
\text { (NCT02091141) }\end{array}$ & 57 & II & Refractory & $\begin{array}{l}\text { HER2 copy number }>6 \text {, or } \\
\text { HER } 2 / C E P 17>2.0 \text {, or IHC } 3+\end{array}$ & Trastuzumab + pertuzumab & 11.5 & 2.9 & $\begin{array}{l}31.6 \text { (39.5 in } \\
\text { KRAS wild-type) }\end{array}$ \\
\hline $\begin{array}{l}\text { TRIUMPH }{ }^{44,45} \\
\text { (UMIN000027887) }\end{array}$ & 17 & II & Refractory & $\begin{array}{l}\text { IHC 3+ or IHC 2+ and } \\
\text { FISH-positive and/or HER2 } \\
\text { amplification in ctDNA }\end{array}$ & Trastuzumab + pertuzumab & NR & 4.0 & 35.3 \\
\hline $\begin{array}{l}\text { MOUNTAINEER }{ }^{46,47} \\
\text { (NCT03043313) }^{\text {NCT3 }}\end{array}$ & 26 & $\|$ & Refractory & $\begin{array}{l}\text { HER2 amplification by NGS, FISH, } \\
\text { or IHC }\end{array}$ & Tucatinib + trastuzumab & 17.3 & 6.2 & 55 \\
\hline $\begin{array}{l}\text { HERACLES-B }{ }^{41,48} \\
\text { (NCT03225937) }\end{array}$ & 30 & $\|$ & $\begin{array}{l}\text { Refractory (after } \\
\text { trastuzumab) }\end{array}$ & $\begin{array}{l}\text { IHC } 3+\text { or IHC } 2+\text { and } \\
\text { FISH-positive }\end{array}$ & Pertuzumab + T-DM1 & NR & 4.8 & 10 \\
\hline $\begin{array}{l}\text { SUMMIT } \\
\text { (NCT0190 } \\
\text { (NC195926) }\end{array}$ & 12 & $\|$ & Second or later & $\begin{array}{l}\text { ERBB2 and/or ERBB3 alterations } \\
\text { identified using NGS }\end{array}$ & Neratinib & NR & NR & 0 \\
\hline $\begin{array}{l}\text { DESTINY-CRC0 }{ }^{51,52} \\
\text { (NCT03384940) }\end{array}$ & 53 & $\|$ & Refractory & $\begin{array}{l}\text { IHC } 3+\text { or IHC } 2+\text { and } \\
\text { FISH-positive }\end{array}$ & Trastuzumab deruxtecan & NR & 6.9 & 45.3 \\
\hline
\end{tabular}

CtDNA = circulating tumour DNA; FISH = fluorescence in situ hybridisation; HER2 = human epidermal growth factor receptor 2; IHC = immunohistochemistry;

$N G S=$ next-generation sequencing; $N R=$ not reported; ORR = objective response rate; $O S=$ overall survival; $P F S=$ progression-free survival; $T$-DM1 = ado-trastuzumab emtansine.

chemotherapy and anti-EGFR therapy. ${ }^{35,41}$ In this study, HER2 positivity was defined as: (1) IHC 3+ (membrane staining in $\geq 50 \%$ of tumour cells); or (2) $I \mathrm{HC} \mathrm{2+}$ and FISH-positive. Among 914 patients with tumours harbouring wild-type KRAS exon 2, 48 patients (5.3\%) were identified as HER2-positive. ${ }^{35}$ A total of 27 patients were enrolled and received trastuzumab and lapatinib therapy. Eight of 27 patients achieved an objective response, including a complete response in one patient. The ORR was $30 \%$, the disease control rate (DCR) was $74 \%$, and median OS was 46 weeks (Table 2) ${ }^{35,41-52}$ Although this was a small, non-randomised, phase ॥ trial, dual HER2-targeted therapy can be expected as a new option for patients with HER2-positive MCRC on the basis of these favourable results.

Subsequently, combination therapy with trastuzumab and pertuzumab was also evaluated in MyPathway, an ongoing phase II basket trial for patients with advanced solid tumours harbouring specific genetic or molecular alterations. ${ }^{42}$ In this trial, patients with HER2-positive solid tumours received treatment with trastuzumab and pertuzumab. For eligibility, HER2 positivity was defined as HER2 copy number $>6$ by next-generation sequencing (NGS) or ISH, HER2/CEP17 ratio >2.0 by FISH, or IHC 3+. A total of 34 patients with HER2-amplified mCRC were enrolled. At a median follow-up of 7.3 months, the ORR was $31.6 \%(18 / 57)$ and the median duration of response was 5.9 months (Table 2). Interestingly, in the wild-type KRAS group only, ORR reached about $40 \%$, which was statistically higher than the ORR (12.5\%) of the mutant KRAS group in this study.

The phase II TRIUMPH study (Multicenter phase II study to evaluate efficacy and safety of combination therapy with trastuzumab and pertuzumab in patients with HER2-positive metastatic colorectal cancer; Clinical trial identification: UMIN000027887) also evaluated combination therapy with trastuzumab and pertuzumab for patients with HER2-positive MCRC.44 Patients with wild-type RAS harbouring HER2-positive mCRC confirmed by IHC/FISH (tissue-positive group) and/or HER2 amplification by a circulating tumour DNA (ctDNA) analysis using Guardant360 assay (ctDNA-positive group), were enrolled. ${ }^{44} \mathrm{~A}$ total of 17 patients received the HER2-targeted therapy with trastuzumab plus pertuzumab every 3 weeks.
In the tissue-positive group, six of 17 patients (35.3\%) achieved objective responses, including one complete response (Table 2). In the ctDNA-positive group, five of 15 patients (33.3\%) achieved an objective response, including one complete response. In an exploratory analysis, five of 11 patients (54.5\%) with wild-type RAS/BRAF/PIK3CA/HER2 by ctDNA analysis at baseline, achieved a confirmed objective response, while none of the five patients with any mutation in any of the four genes at baseline had an objective response. Furthermore, the IHC scores for all patients who achieved an objective response were $3+$, while none of the patients with IHC 2+ had a clinical response. Currently, the S1613 study (Trastuzumab and pertuzumab or cetuximab and irinotecan hydrochloride in treating patients with locally advanced or metastatic HER2/Neu amplified colorectal cancer that cannot be removed by surgery; ClinicalTrials. gov identifier: NCT03365882), a randomised phase II trial to compare the efficacy of trastuzumab and pertuzumab to that of cetuximab and irinotecan for mCRC with HER2 amplification, is ongoing. ${ }^{53,54}$

The efficacy of tucatinib, which is a highly selective oral small molecule TKI of HER2, was evaluated in the MOUNTAINEER study (Tucatinib plus trastuzumab in patients with HER2+ colorectal cancer; ClinicalTrials.gov identifier: NCT03043313).46,47 This was a single-arm phase II trial for patients with HER2-positive, wild-type RAS MCRC who had received standard chemotherapy, including an anti-vascular endothelial growth factor (VEGF) monoclonal antibody. A total of 26 patients were enrolled and received combination therapy with tucatinib and trastuzumab. Among 22 patients who completed $\geq 1$ evaluation, 12 (55\%) achieved an objective response and an additional two showed stable disease over 4 months. The median duration of response was not reached, median OS was 17.3 months, and median PFS was 6.2 months (Table 2).

HERACLES-B (HER2 amplification for colorectal cancer enhanced stratification, cohort B; ClinicalTrials.gov identifier: NCT03225937) is an open-label phase II trial to evaluate the combination of pertuzumab and T-DM1 in untreated wild-type RAS/BRAF and HER2-positive MCRC ( $\mathrm{n}=30$ patients). ${ }^{48}$ The ORR was $10 \%$ and DCR was $80 \%$, with a median PFS of 4.8 months. The primary endpoints, ORR and PFS, were not met (Table 2). 
Table 3: The efficacy of HER2-targeted therapies in biliary tract cancer and pancreatic cancer

\begin{tabular}{|c|c|c|c|c|c|c|c|c|}
\hline Trial & $\mathrm{N}$ & Phase & Line & Definition of HER2 positive & Treatment & OS & PFS & ORR \\
\hline \multicolumn{9}{|c|}{ Biliary tract cancer } \\
\hline $\begin{array}{l}\text { MyPathway }{ }^{43,67} \\
\text { (NCT02091141) }\end{array}$ & 21 & $\|$ & $\begin{array}{l}\text { Second or } \\
\text { third }\end{array}$ & $\begin{array}{l}\text { HER2 copy number }>6 \text {, } \\
\text { HER2/CEP17 }>2.0, \text { IHC } 3+\text {, or } \\
\text { putative activating mutations }\end{array}$ & Trastuzumab + pertuzumab & NR & NR & $19 \%$ \\
\hline $\begin{array}{l}\text { SUMMIT } 49,50 \\
\text { (NCT01953926) }\end{array}$ & 11 & $\|$ & $\begin{array}{l}\text { Second or } \\
\text { later }\end{array}$ & $\begin{array}{l}\text { ERBB2 and/or ERBB3 alterations } \\
\text { identified using NGS }\end{array}$ & Neratinib & NR & NR & $22 \%$ \\
\hline \multicolumn{9}{|c|}{ Pancreatic cancer } \\
\hline Harder et al. ${ }^{68}$ & 17 & $\|$ & First & IHC 3+ or gene amplification & Trastuzumab + capecitabine & 6.9 months & $\begin{array}{l}23.5 \% \text { at } 12 \text { weeks; } \\
11.8 \% \text { at } 6 \text { months }\end{array}$ & NR \\
\hline Safran et al. ${ }^{69}$ & 34 & $\|$ & First & $\mathrm{IHC} \geq 2+$ & Trastuzumab + gemcitabine & $19 \%$ at 1 year & NR & $6 \%$ \\
\hline Safran $\mathrm{H}$ et al. ${ }^{70}$ & 29 & $\|$ & First & $\begin{array}{l}\text { Any patients regardless of } \\
\text { HER2 status }\end{array}$ & Lapatinib + gemcitabine & 4.0 months & NR & $10 \%$ \\
\hline
\end{tabular}

HER2 = human epidermal growth factor receptor 2; IHC = immunohistochemistry; NGS = next-generation sequencing; ORR = objective response rate; OS = overall survival; PFS = progression-free survival

In the SUMMIT study (Neratinib HER mutation basket study; ClinicalTrials.gov identifier NCT01953926), a phase II basket study for HER2-mutant solid tumours, 12 patients with MCRC harbouring HER2 mutation received treatment with neratinib, an irreversible pan-HER TKI.49,50 However, objective response was not observed. Now, the comparison of combination therapy with neratinib plus trastuzumab or neratinib plus cetuximab in patients with KRAS/NRAS/BRAF/PIK3CA wild-type MCRC is being evaluated in a randomised phase II study (ClinicalTrials.gov identifier: NCT03457896). ${ }^{55}$

In the DESTINY-CRC01 trial, a phase $\|$, multicentre, open-label study of T-DXd in patients with HER2-expressing MCRC, a total of 78 patients received T-DXd. They received T-DXd $6.4 \mathrm{mg} / \mathrm{kg}$ every 3 weeks in three cohorts (A: HER2 IHC 3+ or IHC $2+/ \mathrm{ISH}+; \mathrm{B}$ : IHC 2+/ISH-; C. IHC 1+). The primary endpoint was confirmed ORR. In cohort A $(n=53)$, the ORR was $45.3 \%(24 / 53$ pts; $95 \% \mathrm{Cl}$, 31.6-59.6\%) including 1 complete response and 23 partial responses. The median PFS was 6.0 months ( $95 \% \mathrm{Cl}, 4.1$ months-not evaluated); median OS was not reached. No responses were observed in cohorts $B$ $(n=7)$ or $C(n=18))^{51,52}$

In summary, some clinical trials for HER2-positive CRC have shown promising outcomes. However, these trials were performed based on different inclusion criteria and included small number of patients (Table 2). Since the different inclusion criteria make it difficult to interpret the efficacy of HER2-targeted therapy across each trial, an establishment of integrated HER2-positivity criteria is required in CRC.

\section{Oesophageal cancer}

There are two major pathological subtypes of oesophageal cancer, oesophageal squamous cell carcinoma (OSCC) and oesophageal adenocarcinoma (OAC) including GEJ cancer, such as Barrett's adenocarcinoma. The frequency of HER2 amplification is high in OAC, with estimates of approximately $30 \%$ in the Cancer Genome Atlas and $32.2 \%$ in the ToGA study (in GEJ cancer). ${ }^{13,56}$ Trastuzumab plus chemotherapy is recommended in patients with HER2-positive OAC based on the results of TOGA trial. ${ }^{57}$ In OSCC, the frequency of HER2 amplification is much lower than that observed for OAC. In a genomic landscape study in Japan, HER2 amplification was detected in only $2.3 \%$ of OSCC cases. ${ }^{58}$ Another study reported that the frequency of HER2 amplification in
OSCC is $3.9 \%(3 / 76)$, despite a high HER2 amplification rate $(24.0 \%$; 12/50) in GEJ cancer. ${ }^{59}$

In a preclinical study of HER2- and EGFR-overexpressing oesophageal cancer cell lines, trastuzumab and lapatinib inhibited cell growth and enhanced antibody-dependent cytotoxicity. ${ }^{60}$ However, prospective trials of HER2-positive OSCC are lacking to date.

\section{Biliary tract cancer}

In biliary tract cancer (BTC), HER2-targeted therapy has received attention owing to its high frequency of HER2 overexpression. In one study, $54.3 \%$ of BTC cases exhibited HER2 IHC staining (IHC score $\geq 1+$ ), and $10.9 \%$ of BTC cases had an $\mathrm{IHC}$ score of $3+$, although the reported frequency of HER2 amplification is $5-15 \%$ in BTC, particularly in gallbladder cancer. ${ }^{61-64}$ In the Nationwide Cancer Genome Screening Project in Japan, for metastatic BTC (MBTC) samples analysed by NGS, HER2 amplification was detected in three of 92 tumours (3.3\%). ${ }^{65}$ Another comprehensive genomic profiling study of 260 BTCs demonstrated that HER2 mutation was identified in $4-5 \%$, and was mutually exclusive to RAS, BRAF and NF1 mutations. ${ }^{66}$

In the BTC cohort in the MyPathway trial, eight patients with HER2 amplification or HER2 overexpression and three with HER2 mutations (D277Y, S310F, and A775-G776insYVMA) received combination therapy with trastuzumab and pertuzumab. ${ }^{67}$ At a median follow-up of 4.2 months, four patients achieved an objective response (three of eight HER2-amplified/overexpressed patients) and three had stable disease for 4 months (Table 3). ${ }^{43,4,4,50,67-70}$ In the SUMMIT basket study, 11 patients with $\mathrm{mBTC}$ harbouring HER2 mutations received treatment with neratinib, and partial responses were observed in $22 \%$ of the patients. ${ }^{39}$ In addition, the MOSCATO study, a prospective molecular profiling study for advanced cancers, reported that a complete response with trastuzumab plus chemotherapy was achieved in one patient with HER2-positive MBTC. ${ }^{11,72}$ Although sample size of these trials was small, these results suggest that HER2-targeted therapy may also be effective in patients with MBTC harbouring HER2 amplification and HER2-activated alterations.

A single-arm, phase II trial of T-DXd for HER2-positive BTC, the HERB study, is ongoing in Japan (JMA-IIA00423). ${ }^{73}$ In the main cohort, IHC 3+ or IHC 2+ and ISH-positive patients determined by central imaging review are eligible. In an additional exploratory cohort, IHC 2+ and ISH-negative 
Table 4: Ongoing clinical trials of agents targeting HER2

\begin{tabular}{|c|c|c|c|}
\hline Trial & Phase & Line & Treatment \\
\hline \multicolumn{4}{|l|}{ Gastric cancer } \\
\hline PETRARCA (NCT02581462) ${ }^{74}$ & III & $\begin{array}{l}\text { Locally advanced } \\
\text { (perioperative) }\end{array}$ & FLOT \pm trastuzumab + pertuzumab \\
\hline $\begin{array}{l}\text { MK-3475-811/KEYNOTE-811 } \\
(\text { NCT03615326) }\end{array}$ & III & First & Trastuzumab + chemotherapy \pm pembrolizumab \\
\hline MAHOGANY (NCT04082364) ${ }^{76}$ & $\| / I I$ & First & Margetuximab + INCMGA00012 or margetuximab + chemotherapy \pm INCMGA00012 or MGD013 \\
\hline NCT0119169777 & $\|$ & First & Trastuzumab + bevacizumab + chemotherapy \\
\hline NCT01522768 & $\|$ & Second or later & Afatinib + paclitaxel \\
\hline NCT02954536 79 & $\|$ & First & Trastuzumab + pembrolizumab + chemotherapy \\
\hline INTEGA (NCT03409848) ${ }^{80}$ & $\|$ & First & Trastuzumab + nivolumab + ipilimumab or chemotherapy \\
\hline NCT03556345 & $\|$ & Third or later & RC48-ADC \\
\hline NCT0290130182 & $\|$ & First & Trastuzumab + pembrolizumab + capecitabine + cisplatin \\
\hline NCT0427649383 & $\|$ & First & ZW25 + tislelizumab + chemotherapy \\
\hline NCT02689284 $4^{84}$ & $\|$ & Second & Margetuximab + pembrolizumab \\
\hline NCT02795988 & $1 / I 1$ & First & IMU-131(HER-VaxX) \pm chemotherapy \\
\hline NCT03480256 $6^{86}$ & 1 & Refractory & SHR6390 \\
\hline NCT0361968187 & 1 & Refractory & KN026 \\
\hline NCT0114884988 & 1 & Refractory & Margetuximab (MGAH22) \\
\hline NCT03255070 89 & 1 & Refractory & ARX788 \\
\hline NCT0295272990 & 1 & Refractory & XMT-1522 \\
\hline NCT0328472391 & 1 & Refractory & PF-06804103 \\
\hline $\begin{array}{l}\text { DESTINY-Gastric03 } \\
\text { (NCT04379596) }^{92}\end{array}$ & $|/| \mid$ & First or later & Trastuzumab deruxtecan + 5-fluorouracil or capecitabine or duravalumab \\
\hline \multicolumn{4}{|l|}{ Colorectal cancer } \\
\hline $\begin{array}{l}\text { HERACLES-RESCUE } \\
\left(\text { NCT03418558) }{ }^{93}\right.\end{array}$ & $\|$ & Refractory & T-DM1 \\
\hline NCT03457896 55 & $\|$ & Refractory & Neratinib + trastuzumab or neratinib + cetuximab \\
\hline NCT00003995 94 & $\|$ & Second or later & Trastuzumab + irinotecan \\
\hline NCT0384374995 & $\|$ & Second or later & Pyrotinib + trastuzumab \\
\hline NCT0417259796 & II & Refractory & Poziotinib \\
\hline NCT03185988 & II & Second or later & Trastuzumab + irinotecan \\
\hline MODUL (NCT02291289) ${ }^{98}$ & II & First & Capecitabine + trastuzumab + pertuzumab \\
\hline $\mathrm{S} 1613(\mathrm{NCT03365882})^{54}$ & $\|$ & Second or later & Trastuzumab + pertuzumab or cetuximab + irinotecan \\
\hline NCT0422704199 & $|/| \mid$ & Third or later & Pyrotinib + capecitabine \\
\hline \multicolumn{4}{|l|}{ Biliary cancer } \\
\hline BILHER (NCT03613168) & $\|$ & First & Trastuzumab + gemcitabine + cisplatin \\
\hline HERB (JMA-IIA00423) ${ }^{73}$ & $\|$ & Refractory & Trastuzumab deruxtecan \\
\hline NCT03185988101 & $\|$ & Second or later & Trastuzumab + irinotecan or 5-FU or capecitabine \\
\hline \multicolumn{4}{|l|}{ Multiple tumour types } \\
\hline HERALD (JapicCTI-194707) ${ }^{102}$ & $\|$ & Refractory & Trastuzumab deruxtecan \\
\hline NCT02892123 $3^{103}$ & I & Second to fourth & ZW25 \pm chemotherapy \\
\hline NCT03696771 104 & 1 & Refractory & NJH395 \\
\hline NCT03916094 ${ }^{105}$ & 1 & Refractory & $\mathrm{HLX} 22$ \\
\hline NCT03696030 & 1 & Refractory & HER2-CAR T cells \\
\hline VISTA (NCT03740256) $)^{107}$ & 1 & Refractory & HER2-CAR T cells \\
\hline NCT03602079108 & 1 & Refractory & A166 \\
\hline NCT03821233 ${ }^{109}$ & 1 & Refractory & ZW49 \\
\hline NCT03330561 & 1 & Refractory & PRS-343 \\
\hline NCT03650348 & 1 & Refractory & PRS-343 + atezolizumab \\
\hline NCT04278144 ${ }^{112}$ & 1 & Refractory & BDC-1001 \pm pembrolizumab \\
\hline
\end{tabular}

$A D C=$ antibody-drug conjugate; $C A R T$ = chimeric antigen receptor $T ; F L O T=$ fluouracil/leucovorin/oxaliplatin/docetaxel; HER2 = human epidermal growth factor receptor 2; T-DM1 = ado-trastuzumab emtansine. 
patients and IHC 1+ patients are eligible. The primary endpoint of this trial is ORR in the main cohort and the key secondary endpoints are PFS, OS, and ORR in patients with low HER2 expression (Table 4).54,5,73-112

\section{Pancreatic cancer}

The frequency of HER2 overexpression has been reported to be $17-45 \%$ pancreatic cancer, ${ }^{113,114}$ with the frequency of HER2 amplification approximately $3 \% .{ }^{113}$ In both pancreatic-cancer cell lines and a xenograft mouse model, HER2-targeted therapy with trastuzumab showed favourite results. ${ }^{115}$ Therefore, several trials have been performed to clarify the clinical significance of HER2 expression and amplification in patients with metastatic pancreatic cancer and to determine the potential of HER2 as a therapeutic target.

To investigate the efficacy and safety of trastuzumab plus capecitabine as first-line treatment in advanced pancreatic cancer, a prospective, single-arm, open-label, multicentre phase $\|$ trial was conducted. ${ }^{68}$ A total of 207 patients were assessed for HER2 expression and gene amplification in tumour specimens, with 22 and 31 patients diagnosed as HER2 IHC 3+ and 2+ or HER2 amplification, respectively. Among them, $17 \mathrm{IHC} 3+$ or IHC 2+ and ISH-positive patients were enrolled in the study, receiving treatment with trastuzumab and capecitabine. The rate of PFS at 12 weeks was $23.5 \%$, and median OS was 6.9 months. These results were not an improvement over those of standard chemotherapy (Table 3).

In another phase II study of trastuzumab and gemcitabine among 34 patients with metastatic pancreatic cancer, four (12\%) had IHC $3+$ and 30 (88\%) had IHC 2+. ${ }^{69}$ Confirmed partial responses were observed in two patients (6\%) and 13 (41\%) had either an unconfirmed partial response or a $>50 \%$ reduction in CA $19-9$. The median OS for all 34 patients was 7 months, with a 1-year survival rate of 19\% (Table 3). As these results were similar to those for gemcitabine alone, the addition of the anti-HER2 agent does not improve clinical outcomes in pancreatic cancer.

\section{Future perspectives}

The first TOGA trials demonstrated the survival benefit of HER2-targeted therapy for patients with gastric or GEJ cancer with HER2-high expression (IHC 3+ or 2+ and FISH-positive). In the HERACLES trial for HER2-positive MCRC, response rate was higher in patients with IHC 3+ compared with IHC 2+ (44\% versus 14\%) and high HER2 copy number was also related to better treatment outcome. These results suggest that both HER2 amplification and HER2 overexpression are important for selection of patients with $\mathrm{Gl}$ cancer who may benefit from HER2-targeted therapy. Genomic profiling of GI cancers has revealed HER2 somatic mutation is occurring in the absence of amplification. It is unclear whether anti-HER2 antibodies are effective for tumours with HER2 mutation because preclinical studies showed trastuzumab can block cell proliferation and survival in tumour with HER2 mutation, ${ }^{116,117}$ but other studies have shown HER2 mutation weakened the inhibition of trastuzumab. ${ }^{118,119}$ Nevertheless, HER2 somatic mutation is also regarded as a treatment target in some clinical trials because small-molecule inhibitors, such as neratinib, have shown higher effectiveness in blocking the survival of cells expressing HER2 mutants in preclinical studies. ${ }^{116}$ In the SUMMIT study for solid tumour with HER2 mutation, neratinib showed promising tumour responses in particular cancer types, such as BTC.

As we have reviewed above, multiple regimens have been evaluated in GI cancers, but only limited agents have shown clinical benefits due to some potential limitations. First, the frequency of HER2 amplification is normally $<10 \%$ in Gl cancer; except for gastric cancer. ${ }^{34,58,6,1,113}$ To address this issue, broad screening using NGS and several basket trials, such as

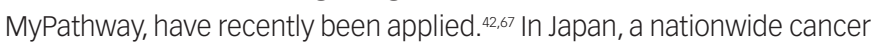
genome screening project, GI-SCREEN, has been in place since 2015, with more than 5,000 genome sequencing results from $\mathrm{Gl}$ cancer tissue samples generated to date. ${ }^{120}$

In addition, ctDNA analysis, a liquid biopsy technology, may be beneficial in a clinical setting given its minimal invasiveness and short turnaround time. ${ }^{121,122}$ In a validation study of 58 patients with metastatic breast cancer, positive and negative predictive value of HER2 amplification in plasma were $70 \%$ and $92 \%$, respectively. ${ }^{23}$ However, in metastatic GI cancer, there are reports of the relationship between HER2 copy number in ctDNA and HER2 overexpression in tissue. An analysis of 60 patients with resectable gastric cancer showed the preoperative plasma HER2 amplification correlated with the tumour HER2 amplification and overexpression $(p<0.001)$, and sensitivity and specificity were $73.3 \%$ and $93.3 \%$, respectively. ${ }^{124}$ In addition, HER2 copy number in CtDNA has been reported to be associated with the efficacy of HER2-targeted therapy in gastric cancer and mCRC. ${ }^{125,126}$ Therefore, ctDNA HER2 analysis may be useful for screening to identify patients with HER2 amplification who may benefit from HER2-targeted therapy.

Based on the GI-SCREEN platform, the GOZILA study (Research on liquid biopsy in patients with gastrointestinal and abdominal malignancies, including colorectal cancer; UMIN000029315) is currently evaluating ctDNA cancer-related genome sequences using Guardant360 for more than 2,000 patients with advanced Gl cancer. ${ }^{127,228}$ Patients can be enrolled in several clinical trials based on the gene alterations identified in GOZILA. For example, HERALD (A basket trial of DS-8201a, a novel HER2-targeted antibody-drug conjugate, for HER2 amplified solid tumors identified by ctDNA analysis; JAPIC ID: JapicCTI-194707) is a basket trial of T-DXd for ctDNA HER2-positive solid tumours, excluding breast, lung, gastric, colorectal, and BTC, based on the GOZILA platform. ${ }^{102}$ Second, a definition of HER2 positivity associated with treatment efficacy has yet to be established for GI cancer, except for gastric cancer. Although established criteria will likely be helpful to improve protocols and interpret results; in the past, clinical trials have been performed with different inclusion criteria by different HER2 testing modalities, such as $\mathrm{IHC}, \mathrm{ISH}, \mathrm{NGS}$, and ctDNA (Table 2). In a recent study spanning Japan, Korea and the USA, the international diagnostic criteria for HER2-amplified mCRC have been harmonised by investigation of the relationship between HER2-IHC/FISH results and HER2 copy number determined by NGS. ${ }^{129}$ In this study, criteria using NGS as well as IHC/FISH have been proposed to identify HER2-positive CRC, which will be validated with additional clinical data in the future.

Currently, several trials involving novel HER2-targeted agents are ongoing in $\mathrm{Gl}$ cancer (Table 4). A phase III trial investigating the efficacy of pembrolizumab, an anti-programmed cell death protein 1 (PD-1) monoclonal antibody, versus placebo in combination with trastuzumab plus chemotherapy in patients with chemotherapy-naive HER2-positive gastric or GEJ cancer is currently ongoing (KEYNOTE-811; ClinicalTrials.gov identifier: NCT03615326). ${ }^{75}$ This is based on the promising results of a phase II trial (ORR of $87 \%$ ), which reported a median PFS of 11.4 months, with a 12 -month OS rate of $76 \% .{ }^{130}$

Margetuximab is an anti-HER2 antibody with increased affinity for both the low-affinity and high-affinity forms of CD16A, an FC receptor. In a first-in-human phase I study, 66 patients with HER2-positive breast, gastric, or other cancers received margetuximab at doses of 
0.1-6.0 mg/kg for 3 of every 4 weeks or once every 3 weeks (10-18 mg/kg). ${ }^{131}$ Among 60 response-evaluable patients, confirmed partial responses were observed in seven (12\%) patients, including two with gastroesophageal cancer, and stable disease was observed in $30(50 \%)$ patients. An ongoing phase $\mathrm{Ib} / \mathrm{ll}$ study is investigating the effects of margetuximab in combination with pembrolizumab in patients with HER2-positive gastric or GEJ cancers previously treated with trastuzumab. In the preliminary results of 92 patients, ORR was $22.4 \%$, DCR was $57.7 \%$, median PFS was 2.7 months, and median OS was 13.9 months (ClinicalTrial.gov identifier: NCT02689284). ${ }^{132}$

ZW25 is a bispecific antibody that simultaneously binds to two epitopes on HER2: one in extracellular domain 4, containing the trastuzumab binding site, and the other in extracellular domain 2, containing the pertuzumab binding site. Preclinical studies using this agent have demonstrated high levels of anti-tumour activity at a wide range of HER2 expression levels, and that ZW25 is a more effective inhibitor of HER2 signalling than the combination of trastuzumab and pertuzumab because of its improved binding, clustering, and receptor internalisation and downregulation. ${ }^{133,134}$ In a phase I study in HER2-expressing solid tumours, ZW25 was well tolerated and demonstrated ORR of $67 \%$ in BTC, $>30 \%$ in CRC and gastric or GEJ cancer. ${ }^{135}$

NJH395 is a bispecific antibody derivative, which is animmune-stimulating antibody conjugate consisting of a monoclonal anti-HER2 antibody conjugated to an immunostimulatory agent. ${ }^{136}$
A phase I trial designed to determine safety and dose of NJH395 is currently recruiting patients with non-breast HER2-positive advanced-stage cancer (ClinicalTrials.gov identifier: NCT03696771). ${ }^{104}$ 4-1BB (CD137) is a key costimulatory immunoreceptor and promising therapeutic target in cancer. PRS-343, which is a $4-1 \mathrm{BB} / \mathrm{HER} 2$ bispecific molecule, is designed to facilitate T-cell costimulation by tumour-localized, HER2-dependent 4-1BB clustering and activation. ${ }^{137}$ In a phase I, dose-escalation study, PRS-343 monotherapy was well-tolerated in all doses and demonstrated objective response in two of 18 evaluable patients (11\%) in heavily pre-treated patient with HER2-positive solid tumours. ${ }^{138}$ Currently, another phase I trial with PRS-343 in combination with atezolizumab in HER2-positive solid tumours, is ongoing (ClinicalTrials.gov identifier: NCT03650348). ${ }^{137}$

\section{Conclusion}

In GI cancers, HER2-targeted therapy is an area of active investigation. Although trastuzumab in advanced gastric or GEJ cancer is the only anti-HER2 agent in GI cancer so far, other novel HER2-targeted agents have emerged and shown promising efficacy in gastric and other Gl cancers. Although several issues, including more precise selection of patients to receive HER2-targeted therapies, still need to be addressed for progress in the development of HER2-targeted therapy in Gl cancer, novel methods such as screening using ctDNA, basket trials, and an establishment of international diagnostic criteria will likely enable continued advances in HER2-targeted therapy in the future. $\square$
Yarden Y, Sliwkowski MX. Untangling the ErbB signalling network. Nat Rev Mol Cell Biol. 2001;2:127-37.

2 . Pinkas-Kramarski R, Soussan L, Waterman $H$, et al. Diversification of Neu differentiation factor and epidermal growth factor signalling by combinatorial receptor interactions. EMBO I 1996:15:2452-67.

3. Gutierrez C, Schiff R. HER2: biology, detection, and clinical implications. Arch Pathol Lab Med. 2011;135:55-62.

4. Slamon DJ, Clark GM, Wong SG, et al. Human breast cancer: correlation of relapse and survival with amplification of the HER-2/neu oncogene. Science. 1987;235:177-82.

5. Slamon DJ, Leyland-Jones B, Shak S, et al. Use of chemotherapy plus a monoclonal antibody against HER2 for metastatic breast cancer that overexpresses HER2. N Engl I Med. 2001;344:783-92.

6. Modi S, Saura C, Yamashita T, et al. Trastuzumab deruxtecan in previously treated HER2-positive breast cancer. N Eng/ J Med. 2020:382:610-21

7. Murthy RK, Loi S, Okines A, et al. Tucatinib, trastuzumab, and capecitabine for HER2-positive metastatic breast cancer. N Engl I Med. 2020:382:597-609.

8. Rugo HS, Im SA, Cardoso F, et al. Phase 3 SOPHIA study of margetuximab + chemotherapy vs trastuzumab + chemotherapy in patients with HER2 + metastatic breast cance after prior anti-HER2 therapies: second interim overall survival analysis. Cancer Res. 2020;80(Suppl. 4):GS-02.

9. Ogitani Y, Aida T, Hagihara K, et al. DS-8201a, A novel HER2-targeting ADC with a novel DNA topoisomerase inhibitor, demonstrates a promising antitumor efficacy with differentiation from T-DM1. Clin Cancer Res. 2016;22:5097-108.

10. Park DI, Yun JW, Park JH, et al. HER-2/neu amplification is an independent prognostic factor in gastric cancer. Dig Dis SCi. 2006;51:1371-9.

11. Yano $T$, Doi T, Ohtsu A, et al. Comparison of HER2 gene amplification assessed by fluorescence in situ hybridization and HER2 protein expression assessed by immunohistochemistry in gastric cancer. Oncol Rep. 2006;15:65-71.

12. Giuffre $G$, leni $A$, Barresi $V$, et al. HER2 status in unusual histological variants of gastric adenocarcinomas. J Clin Pathol. 2012;65:237-41

13. Van Cutsem E, Bang YJ, Feng-Yi F, et al. HER2 screening data from TOGA: targeting HER2 in gastric and gastroesophageal junction cancer. Gastric Cancer. 2015;18:476-84.

14. Grabsch $\mathrm{H}$, Sivakumar S, Gray S, et al. HER2 expression in gastric cancer. Rare, heterogeneous and of no prognostic value - conclusions from 924 cases of two independent series. Cell Oncol. 2010;32:57-65.

15. Lee HE, Park KU, Yoo SB, et al. Clinical significance of intratumoral HER2 heterogeneity in gastric cancer. Eur $J$ Cancer 2013:49:1448-57

16. Bang YJ, Van Cutsem E, Feyereislova A, et al. Trastuzumab in combination with chemotherapy versus chemotherapy alone for treatment of HER2-positive advanced gastric or gastro-oesophageal junction cancer (ToGA): a phase 3 open-label, randomised controlled trial. Lancet. 2010;376:687-97.

17. Bartley AN, Washington MK, Colasacco C, et al. HER2 testing and clinical decision making in gastroesophageal adenocarcinoma: guideline from the college of American Pathologists, American Society for Clinical Pathology, and the American Society of Clinical Oncology. J Clin Oncol 2017:35:446-64.

18. ClinicalTrials gev TOGA study - A study of herceptin (trastuzumab) in combination with chemotherapy compare with chemotherapy alone in patients with HER2-positive advanced gastric cancer. Available at: https://clinicaltrials.gov/ ct2/show/NCT01041404 (accessed 28 July 2020)

19. Makiyama A, Sagara K, Kawada J, et al. A randomized phase II study of weekly paclitaxel \pm trastuzumab in patients with HER2-positive advanced gastric or gastro-oesophageal junction cancer refractory to trastuzumab combined with fluoropyrimidine and platinum:WJOG7112G (T-ACT). J Clin Oncol. 2018;36(Suppl. 15):4011.

20. Hecht JR, Bang YJ, Qin SK, et al. Lapatinib in combination with capecitabine plus oxaliplatin in human epidermal growth factor receptor 2-Positive advanced or metastatic gastric, oesophageal, or gastroesophageal adenocarcinoma TRIO-013/LOGiC-a randomized phase III trial. J Clin Oncol. 2016:34:443-51.

21. ClinicalTrials.gov. LOGiC - lapatinib optimization study in ErbB2 (HER2) positive gastric cancer: a phase III global, blinded study designed to evaluate clinical endpoints and safety of chemotherapy plus lapatinib. Available at: https://clinicaltrials. gov/ct2/show/NCT00680901 (accessed 28 July 2020).

22. Satoh $\mathrm{T}$, Xu RH, Chung $\mathrm{HC}$, et al. Lapatinib plus paclitaxel versus paclitaxel alone in the second-line treatment of HER2-amplified advanced gastric cancer in Asian populations: TyTAN-a randomized, phase III study. J Clin Oncol. 2014:32:2039-49.

23. ClinicalTrials gov Lapatinib in combination with weekly paclitaxel in patients with ErbB2 amplified advanced gastric cancer. Available at: https://clinicaltrials.gov/ct2/show/ NCT00486954 (accessed 28 July 2020).

24. Thuss-Patience PC Shah MA Ohtsu A et al Trastuzumab emtansine versus taxane use for previously treated HER2-positive locally advanced or metastatic gastric or gastro-oesophageal junction adenocarcinoma (GATSBY): an international randomised, open-label, adaptive, phase 2/3 study. Lancet Oncol. 2017;18:640-53.

25. ClinicalTrials.gov. A study of trastuzumab emtansine versus taxane in participants with human epidermal growth factor receptor 2 (HER2)-positive advanced gastric cancer. Available at: https://clinicaltrials.gov/ct2/show/NCT01641939 (accessed 28 July 2020)

26. Tabernero J, Hoff PM, Shen L, et al. Pertuzumab plus trastuzumab and chemotherapy for HER2-positive metastatic gastric or gastro-oesophageal junction cancer (JACOB): final analysis of a double-blind, randomised, placebo-controlled analysis of a double-blind, randomised, placebo-c 3 study Lancet Oncol. 2018:19:1372-84.

27. ClinicalTrials.gov. A study of pertuzumab in combination with trastuzumab and chemotherapy in participants with human epidermal growth factor receptor 2 (HER2)-positive metastatic gastroesophageal junction or gastric cancer (JACOB). Available at: https://clinicaltrials.gov/ct2/show/NCT01774786 (accessed 28 July 2020).

28. Shitara K, Bang YJ, Iwasa S, et al. Trastuzumab deruxtecan in previously treated HER2-positive gastric cancer. N Eng/ J Med.
2020;382:2419-30

29. ClinicalTrials.gov. DS-8201a in human epidermal growth factor receptor 2 (HER2)-expressing gastric cancer [DESTINY-Gastric01]. Available at: https://clinicaltrials.gov/ct2/ show/NCT03329690 (accessed 28 July 2020).

30. Diéras V, Miles D, Verma S, et al. Trastuzumab emtansine versus capecitabine plus lapatinib in patients with previously treated HER2-positive advanced breast cancer (EMILIA): A descriptive analysis of final overall survival results from a randomised, open-label, phase 3 trial. Lancet Oncol. 2017;18:732-42.

31. Ishimine $Y$, Goto $A$, Watanabe $Y$, et al. Loss of HER2 positivity after trastuzumab in HER2-positive gastric cancer: is change in HER2 status significantly frequent? Case Rep Gastrointest Med. 2015;2015:132030

32. Swain SM, Kim SB, Cortés J, et al. Pertuzumab, trastuzumab, and docetaxel for HER2-positive metastatic breast cancer (CLEOPATRA study): overall survival results from a randomised, double-blind, placebo-controlled, phase 3 study. Lancet Oncol. 2013:14:461-71.

33. ClinicalTrials.gov. A study to evaluate pertuzumab + trastuzumab + docetaxel vs. placebo + trastuzumab + docetaxel in previously untreated HER2-positive metastatic breast cancer (CLEOPATRA). Available at: https://clinicaltrials gov/ct2/show/NCT00567190 (accessed 28 July 2020).

34. Martin V, Landi L, Molinari F, et al. HER2 gene copy number status may influence clinical efficacy to anti-EGFR monoclonal antibodies in metastatic colorectal cancer patients. Br I Cancer. 2013;108:668-75.

35. Sartore-Bianchi A, Trusolino L, Martino C, et al. Dual-targeted therapy with trastuzumab and lapatinib in treatment-refractory, KRAS codon 12/13 wild-type, HER2-positive metastatic colorectal cancer (HERACLES): a proof-of-concept, multicentre, open-label, phase 2 trial. Lancet Oncol. 2016;17:738-46.

Ingold Heppner B, Behrens HM, Balschun K, et al. HER2/neu testing in primary colorectal carcinoma. Br $\lrcorner$ Cancer. 2014;111:1977-84.

37. Richman SD, Southward K, Chambers P, et al. HER2 overexpression and amplification as a potential therapeutic target in colorectal cancer: analysis of 3256 patients enrolled in the QUASAR, FOCUS and PICCOLO colorectal cancer trials. J Pathol. 2016;238:562-70.

38. Jeong JH, Kim J, Hong YS, et al. HER2 Amplification and cetuximab efficacy in patients with metastatic colorectal cancer harboring wild-type RAS and BRAF. Clin colorectal Cancer. 2017;16:e147-52

39. Sawada K, Nakamura Y, Yamanaka T, et al. Prognostic and predictive value of HER2 amplification in patients with metastatic colorectal cancer. Clin Colorectal Cancer. 2018;17:198-205.

40. Bertotti A, Migliardi G, Galimi F, et al. A molecularly annotated platform of patient-derived xenografts ("xenopatients") identifies HER2 as an effective therapeutic target in cetuximab-resistant colorectal cancer. Cancer Discov. 2011;1:508-23.

41. ClinicalTrials.gov. Evaluation of trastuzumab in combination with lapatinib or pertuzumab in combination with trastuzumab-emtansine to treat patients with HER2-positive metastatic colorectal cancer (HERACLES). Available at: 
https://clinicaltrials.gov/ct2/show/NCT03225937 (accessed 28 July 2020).

42. Meric-Bernstam F, Hurwitz H, Raghav KPS, et al. Pertuzumab plus trastuzumab for HER2-amplified metastatic colorectal cancer (MyPathway): an updated report from a multicentre, open-label, phase 2a, multiple basket study. Lancet Oncol. 2019;20:518-30.

43. ClinicalTrials.gov. My Pathway: A study evaluating herceptin/ perjeta, tarceva, zelboraf/cotellic, erivedge, alecensa, and tecentriq treatment targeted against certain molecular alterations in participants with advanced solid tumors. Available at: https://clinicaltrials.gov/ct2/show/NCT0209114 (accessed 28 July 2020)

44. Nakamura Y, Okamoto W, Kato T, et al. TRIUMPH: Primary efficacy of a phase II trial of trastuzumab (T) and pertuzumab (P) in patients (pts) with metastatic colorectal cancer (MCRC) with HER2 (ERBB2) amplification (amp) in tumour tissue or circulating tumour DNA (ctDNA): A GOZILA sub-study. Ann Oncol. 2019;30(Suppl. 5):v199-200.

45. UMIN-CTR Clinical Trial. Multicenter phase II study to evaluate efficacy and safety of combination therapy with trastuzumab and pertuzumab in patients with HER2-positive metastatic colorectal cancer. Available at: https:// upload.umin.ac.jp/cgiopen-bin/ctr_e/ctr_view.cgi?recptno=R000031949 (accesse 28 July 2020)

46. Strickler JH, Zemla T, Ou F, et al. Trastuzumab and tucatinib fo the treatment of HER2 amplified metastatic colorectal cance (mCRC): Initial results from the MOUNTAINEER trial. Ann Oncol. 2019;30(Suppl. 5):v200

47. ClinicalTrials.gov. Tucatinib plus trastuzumab in patients with HER2+ colorectal cancer. Available at: https://clinicaltrials.gov/ ct2/show/NCT03043313 (accessed 28 July 2020).

48. Sartore-Bianchi A, Martino C, Lonardi S, et al. Phase II study of pertuzumab and trastuzumab-emtansine (T-DM1) in patients with HER2-positive metastatic colorectal cancer: The HERACLES-B (HER2 Amplification for Colo-rectaL cancer Enhanced Stratification, cohort B) trial. Ann Oncol. cancer Enhanced Stratification,

49. Hyman DM, Piha-Paul SA, Won $\mathrm{H}$, et al. HER kinase inhibition in patients with HER2- and HER3-mutant cancers. Nature. 2018;554:189-94

50. ClinicalTrials.gov. Neratinib HER mutation basket study (SUMMIT). Available at: https://clinicaltrials.gov/ct2/show/ NCT01953926 (accessed 28 July 2020)

51. Siena S, Di Bartolomeo M, Raghav KPS, et al. A phase II, multicenter, open-label study of trastuzumab deruxtecan (T-DXd; DS-8201) in patients (pts) with HER2-expressing metastatic colorectal cancer (mCRC): DESTINY-CRCO1. J Clin Oncol. 2020;(Suppl. 15):4000.

52. ClinicalTrials.gov. DS-8201a in human epidermal growth facto receptor2 (HER2)-expressing colorectal cancer (DESTINY-CRC01). Available at: https://clinicaltrials.gov/ct2/ (DESTINY-CRC01). Available at: httpS://clinical

53. Raghav KPS, MCDonough S, Tan BR, et al. A randomized phase I study of trastuzumab and pertuzumab (TP) compared to cetuximab and irinotecan (CETIRI) in advanced/metastatic colorectal cancer (mCRC) with HER2 amplification: S1613. $J$ Clin Oncol. 2018;36(Suppl. 15):TPS3620.

54. ClinicalTrials.gov. S1613, trastuzumab and pertuzumab or cetuximab and irinotecan hydrochloride in treating patients with locally advanced or metastatic HER2/neu amplified colorectal cancer that cannot be removed by surgery. Available at: https://clinicaltrials.gov/ct2/show/NCT03365882 (accessed 28 July 2020).

55. ClinicalTrials.gov. Study of neratinib +trastuzumab or neratinib + cetuximab in patients with KRAS/NRAS/BRAF/PIK3CA
wild-type metastatic colorectal cancer by HER2 status. Available at: https://clinicaltrials.gov/ct2/show/NCT03457896 Available at: https://clinicat
(accessed 28 July 2020)

56. Cancer Genome Atlas Research Network; Analysis Working Group. Integrated genomic characterization of oesophageal carcinoma. Nature. 2017;541:169-75.

57. Muro K, Lordick F, Tsushima T, et al. Pan-Asian adapted ESMO Clinical Practice Guidelines for the management of patients with metastatic oesophageal cancer: a JSMO-ESMO initiative endorsed by CSCO, KSMO, MOS, SSO and TOS. Ann Oncol 2019;30:34-43.

58. Sawada G, Niida A, Uchi R, et al. Genomic landscape of oesophageal squamous cell carcinoma in a Japanese population. Gastroenterology. 2016;150:1171-82.

59. Huang JX, Zhao K, Lin M, et al. HER2 gene amplification in oesophageal squamous cell carcinoma is less than in gastroesophageal junction and gastric adenocarcinoma. gastroesophageal junction

60. Mimura K, Kono K, Maruyama T, et al. Lapatinib inhibits receptor phosphorylation and cell growth and enhances antibody-dependent cellular cytotoxicity of EGFR- and HER2-overexpressing oesophageal cancer cell lines. Int J Cancer. 2011;129:2408-16.

61. Nam A-R, Kim J-W, Cha Y, et al. Therapeutic implication of HER2 in advanced biliary tract cancer. Oncotarget. 2016;7:58007-21.

62. Harder J, Waiz O, Otto F, et al. EGFR and HER2 expression in advanced biliary tract cancer. World I Gastroenterol. 2009;15:4511-7.

63. Shafizadeh N, Grenert JP, Sahai V, et al. Epidermal growth factor receptor and HER-2/neu status by immunohistochemistry and fluorescence in situ hybridization in adenocarcinomas of the biliary tree and gallbladder. Hum Pathol. 2010;41:485-92.

64. Nakazawa K, Dobashi Y, Suzuki S, et al. Amplification and overexpression of c-erbB-2, epidermal growth factor receptor, and c-met in biliary tract cancers. J Pathol. 2005;206:356-65.

65. Morizane C, Komatsu Y, Takahashi H, et al. The nationwid cancer genome screening project in Japan, SCRUM Japan GISCREEN: Efficient identification of cancer
in advanced biliary tract cancer. Ann Oncol.
2017;28(Suppl. 5):V244

66. Nakamura H, Arai Y, Totoki Y, et al. Genomic spectra of biliary tract cancer. Nat Genet. 2015;47:1003-10.

67. Javle MM, Hainsworth JD, Swanton C, et al. Pertuzumab trastuzumab for HER2-positive metastatic biliary cancer: preliminary data from MyPathway. I Clin Oncol. 2017;35(Suppl. 4):402.

68. Harder J, Ihorst $\mathrm{G}$, Heinemann V, et al. Multicentre phase II trial of trastuzumab and capecitabine in patients with HER2 overexpressing metastatic pancreatic cancer. Br J Cancer. 2012;106:1033-8.

69. Safran $\mathrm{H}$, lannitti $\mathrm{D}$, Ramanathan $\mathrm{R}$, et al. Herceptin and gemcitabine for metastatic pancreatic cancers that overexpress HER-2/neu. Cancer Invest. 2004;22:706-12.

70. Safran $\mathrm{H}$, Miner T, Bahary N, et al. Lapatinib and gemcitabine for metastatic pancr

71. Massard C, Michiels S, Ferté C, et al. High-throughput genomics and clinical outcome in hard-to-treat advanced cancers: results of the MOSCATO 01 trial. Cancer Discov. 2017:7:586-95.

72. ClinicalTrials.gov. Molecular screening for cancer treatment optimization (MOSCATO 02). Available at: https://clinicaltrials. gov/ct2/show/NCT01566019 (accessed 28 July 2020).

3. NIPH Clinical Trials. A phase II trial of DS-8201a for HER2 positive biliary tract cancer. Available at: https://rctportal.niph go.jp/en/detail?trial_id=JMA-IIA00423 (accessed 28 July 2020).

74. ClinicalTrials.gov. FLOT vs. FLOT/herceptin/pertuzumab for perioperative therapy of HER-2 expressing gastric or GEJ cancer (PETRARCA). Available at: https://clinicaltrials.gov/ct2/ show/NCT02581462 (accessed 28 July 2020).

75. ClinicalTrials.gov. Pembrolizumab/placebo plus trastuzumab plus chemotherapy in human epidermal growth factor recepto 2 positive (HER2+) advanced gastric or gastroesophageal junction (GEJ) adenocarcinoma (MK-3475-811/KEYNOTE-811). Available at: https://clinicaltrials.gov/ct2/show/NCT03615326 (accessed 28 July 2020)

76. ClinicalTrials gov Combination margetuximab, INCMGA00012, MGD013, and chemotherapy phase 2/3 trial in HER2+ gastric/ GEJ cancer (MAHOGANY). Available at: https://clinicaltrials.gov/ ct2/show/NCT04082364 (accessed 28 July 2020).

77. ClinicalTrials.gov. CAPOX, bevacizumab and trastuzumab for patients with HER2-positive metastatic esophagogastric cancer. Available at: https://clinicaltrials.gov/ct2/show/NCT01191697 (accessed 28 July 2020)

78. ClinicalTrials.gov. Afatinib and paclitaxel in patients with advanced HER2-positive trastuzumab-refractory advanced esophagogastric cancer. Available at: https://clinicaltrials.gov/ ct2/show/NCT01522768 (accessed 28 July 2020).

79. ClinicalTrials. gov. Phase II trial of pembrolizumab with trastuzumab and chemotherapy in advanced HER2 positive esophagogastric (EG) cancer. Available at: https://clinicaltrials gov/ct2/show/NCT02954536 (accessed 28 July 2020).

80. ClinicalTrials. gov. Ipilimumab or FOLFOX in combination with nivolumab and trastuzumab in HER2 positive esophagogastric adenocarcinoma (INTEGA). Available at: https://clinicaltrials. gov/ct2/show/NCT03409848 (accessed 28 July 2020).

81. ClinicalTrials.gov. A study of RC48-ADC in local advanced or metastatic gastric cancer subjects with the overexpression of HER2. Available at: https://clinicaltrials.gov/ct2/show/ NCT03556345 (accessed 28 July 2020).

82. ClinicalTrials.gov Pembrolizumab, trastuzumab, HER2 positive gastric cancer. Available at: https://clinicaltrials.gov/ct2/show/ NCT02901301 (accessed 28 July 2020).

83. ClinicalTrials. gov. Anti-HER2 bispecific antibody zw25 activity in combination with chemotherapy with/without tislelizumab. Available at: https://clinicaltrials.gov/ct2/show/NCT04276493 (accessed 28 July 2020)

84. ClinicalTrials gov Combination margetuximab and pembrolizumab for advanced, metastatic HER2(+) gastric or gastroesophageal junction cancer. Available at: https://clinicaltrials.gov/ct2/show/NCT02689284 (accessed 28 July 2020)

85. ClinicalTrials.gov. A study of IMU-131(HER-Vaxx) and chemotherapy compared to chemotherapy only in patients with HER2 positive advanced gastric cancer. Available at: https://clinicaltrials.gov/ct2/show/NCT02795988 (accessed 28 July 2020)

86. ClinicalTrials.gov. Study to evaluate SHR6390 combined with pyrotinib in patients with HER2 positive gastric cancer. Available at: https://clinicaltrials.gov/ct2/show/NCT03480256 (accessed 28 July 2020).

87. ClinicalTrials gov Trial of KNO26 in patients with HER2-positive advanced malignant breast cancer and gastric cancer. Available at: https://clinicaltrials.gov/ct2/show/NCT03619681 (accessed 28 July 2020)

88. ClinicalTrials.gov. Safety study of MGAH22 in HER2-positive carcinomas. Available at: https://clinicaltrials.gov/ct2/show/ NCT01148849 (accessed 28 July 2020).

89. ClinicalTrials.gov. A dose-escalation study of ARX788, IV administered in subjects with advanced cancers with HER2 expression. Available at: https://clinicaltrials.gov/ct2/show/ NCT03255070 (accessed 28 July 2020).

90. ClinicalTrials.gov. Study of antibody drug conjugate in patients with advanced breast cancer expressing HER2. Available at: https://clinicaltrials.gov/ct2/show/NCT02952729 (accessed 28 July 2020).

91. ClinicalTrials.gOv. PF-06804103 dose escalation in HER2 positive solid tumors. Available at: https://clinicaltrials.gov/ct2/show/ NCT03284723 (accessed 28 July 2020).

92. ClinicalTrials.gov. Ph1b/2 study of the safety and efficacy of T-DXd combinations in advanced HER2+ gastric cancer (DESTINY-Gastric03) (DG-03). Available at: https://clinicaltrials. gov/ct2/show/NCT04379596 (accessed 28 July 2020).

93. ClinicalTrials.gov. Study of trastuzumab-emtansine in patients

after trastuzumab and lapatinib. (RESCUE). Available at: https:// clinicaltrials.gov/ct2/show/NCT03418558 (accessed 28 July 2020)

94. ClinicalTrials.gov. Monoclonal antibody plus chemotherapy in treating patients with advanced colorectal cancer that overexpresses HER2. Available at: https://clinicaltrials.gov/ct2/ show/NCT00003995 (accessed 28 July 2020).

95. ClinicalTrials.gov. Pyrotinib in combination with trastuzumab in treatment-refractory, HER2-positive metastatic colorectal cancar. Available at: https://clinicaltrials.gov/ct2/show/ NCT03843749 (accessed 28 July 2020)

96. ClinicalTrials.gov. A study of poziotinib in patients with EGFR or HER2 activating mutations in advanced malignancies. Available at: https://clinicaltrials.gov/ct2/show/NCT04172597 (accessed 28 July 2020)

97. ClinicalTrials.gov. Anti-HER2 therapy in patients of HER2 positive metastatic carcinoma of digestive system. Available at: https://clinicaltrials.gov/ct2/show/NCT03185988 (accessed 28 July 2020).

98. ClinicalTrials.gov. A study of biomarker-driven therapy in metastatic colorectal cancer (MCRC) (MODUL). Available at: https://clinicaltrials.gov/ct2/show/NCTO2291289 (accessed 28 July 2020).

99. ClinicalTrials.gov. A study of pyrotinib combined with capecitabine for metastatic HER-2 positive colorectal cancer. Available at: https://clinicaltrials.gov/ct2/show/NCT0422704 (accessed 28 July 2020)

100. ClinicalTrials.gov. Trastuzumab in HER2-positive biliary tract cancer (BILHER). Available at: https://clinicaltrials.gov/ct2/ show/NCT03613168 (accessed 28 July 2020).

101. ClinicalTrials.gov. Anti-HER2 therapy in patients of HER2 positive metastatic carcinoma of digestive system. Available at: https://clinicaltrials.gov/ct2/show/NCT03185988 (accessed 28 July 2020)

102. NIPH Clinical Trials. HERALD study. Available at: https://rctportal. niph.go.jp/en/detail?trial_id=JapicCTI-194707 (accessed 28 July 2020).

103. ClinicalTrials.gov. Trial of ZW25 in patients with advanced HER2 expressing cancers. Available at: https://clinicaltrials.gov/ct2/ show/NCT02892123 (accessed 28 July 2020).

104. ClinicalTrials.gov. Study to determine safety and dose of NJH395 in non-breast HER2+ advanced cancer. Available at: https://clinicaltrials.gov/ct2/show/NCT03696771 (accessed 28 July 2020)

105. ClinicalTrials.gov. Evaluate safety, tolerability and pharmacokinetics of HLX22 in patients with advanced solid tumors overexpressing HER2. Available at: https://clinicaltrials. gov/ct2/show/NCT03916094 (accessed 28 July 2020)

106. ClinicalTrials.gov. HER2-CAR T cells in treating patients with recurrent brain or leptomeningeal metastases. Available at: https://clinicaltrials.gov/ct2/show/NCT03696030 (accessed 28 July 2020).

107. ClinicalTrials.gov. Binary oncolytic adenovirus in combination with HER2-specific autologous CAR VST, advanced HER2 positive solid tumors (VISTA). Available at: https://clinicaltrials gov/ct2/show/NCT03740256 (accessed 28 July 2020).

08. ClinicalTrials.gov. Study of A166 in patients with relapsed/ refractory cancers expressing HER2 antigen or having amplifie HER2 gene. Available at: https://clinicaltrials.gov/ct2/show/ NCT03602079 (accessed 28 July 2020)

109. ClinicalTrials. gov. A dose finding study of ZW49 in patients with HER2-positive cancers. Available at: https://clinicaltrials.gov/ ct2/show/NCT03821233 (accessed 28 July 2020).

110. ClinicalTrials.gov. PRS-343 in HER2-positive solid tumors. Available at: https://clinicaltrials.gov/ct2/show/NCT03330561 (accessed 28 July 2020),

111. ClinicalTrials gov PRS-343 in combination with atezolizumab in HER2-positive solid tumors. Available at: https://clinicaltrials. gov/ct2/show/NCT03650348 (accessed 28 July 2020)

112. ClinicalTrials.gov. A first-in-human study using BDC-1001 in advanced and HER2-expressing solid tumors. Available at: https://clinicaltrials.gov/ct2/show/NCT04278144 (accessed 28 July 2020)

113. Assenat E, Azria D, Mollevi C, et al. Assessment of HER-2 status in pancreatic adenocarcinoma: correlation of immunohistochemistry, quantitative real-time RT-PCR, and FISH with aneuploidy and survival. Oncotarget. 2015;6:12796-808.

114. Yamanaka Y, Friess H, Kobrin MS, et al. Overexpression of HER2/neu oncogene in human pancreatic carcinoma. Hum Pathol. 1993:24:1127-34.

115. Büchler P, Reber HA, Eibl G, et al. Combination therapy for advanced pancreatic cancer using Herceptin plus chemotherapy Int I Oncol. 2005:27:1125-30.

116. Pahuja KB, Nguyen TT, Jaiswal BS, et al. Actionable activating oncogenic ERBB2/HER2 transmembrane and juxtamembrane domain mutations. Cancer Cell. 2018;34:792-806. 
123. Gevensleben H, Garcia-Murillas I, Graeser MK, et al. Noninvasive detection of HER2 amplification with plasma DNA digital PCR. Clin Cancer Res. 2013;19:3276-84.

124. Shoda K, Ichikawa D, Fujita Y, et al. Monitoring the HER2 copy number status in circulating tumour DNA by droplet digital PC in patients with gastric cancer. Gastric Cancer. 2017;20:126-35

125. Wang H, Li B, Liu Z, et al. HER2 copy number of circulating tumour DNA functions as a biomarker to predict and monitor trastuzumab efficacy in advanced gastric cancer. Eur $J$ Cancer. 2018;88:92-100.

126. Siravegna G, Sartore-Bianchi A, Nagy RJ, et al. Plasma HER2 (ERBB2) copy number predicts response to HER2-targeted (ERBB2) COpy number predicts response to HER2-targeted
therapy in metastatic colorectal cancer. Clin Cancer Res. therapy in metastatic

127. Nakamura $Y$, Taniguchi $H$, Bando $H$, et al. Utility of circulating tumour DNA (ctDNA) versus tumour tissue clinical sequencing for enrolling patients (Pts) with advanced gastrointestinal (GI) cancer to matched clinical trials: SCRUM-Japan GI-SCREEN and GOZILA combined analysis. J Clin Oncol. 2020;(Suppl. 5):5.

128. UMIN-CTR Clinical Trial. Research on liquid biopsy in patients with gastrointestinal and abdominal malignancies, including colorectal cancer. Available at: https://upload.umin.ac.jp/cgi-
open-bin/ctr_e/ctr_view.cgi?recptno=R000033509 (accessed 28 July 2020)

129. Fujii S, Magliocco AM, Kim J, et al. international harmonization of provisional diagnostic criteria for ERBB2-amplified metastatic colorectal cancer allowing for screening by next-generation sequencing panel. JCO Precis Oncol. 2020;4:6-19.

130. Janjigian YY, Maron S, Chou JF, et al. First-line pembrolizumab (P), trastuzumab (T), capecitabine (C) and oxaliplatin (O) in HER2-positive metastatic esophagogastric adenocarcinoma. Ann Oncol. 2019;(Suppl. 5):v253-324.

131. Bang YJ, Giaccone G, Im SA, et al. First-in-human phase 1 study of margetuximab (MGAH22), an Fc-modified chimeric monoclonal antibody, in patients with HER2-positive advance
solid tumors. Ann Oncol. 2017;28:855-61.

132. Catenacci DVT, Lim KH, Uronis HE, et al. Antitumor activity of margetuximab (M) plus pembrolizumab (P) in patients (pts) with advanced HER2+ (IHC3+) gastric carcinoma (GC) J Clin Oncol. 2019;(Suppl. 4):65

133. ZW25 effective in HER2-positive cancers. Cancer Discov. 2019;9:8.

134. Hausman DF, Hamilton EP, Beeram M, et al. Phase 1 study of ZW25, a bispecific anti-HER2 antibody, in patients with advanced HER2-expressing cancers. I Clin Oncol.
2017;35(Suppl. 4):TPS215

135. Meric-Bernstam F, Hanna D, Beeram M, et al. Safety, anti-tumor activity, and biomarker results of the HER2-targeted bispecific antibody ZW25 in HER2-expressing solid tumours. Ann Oncol. 2019;(Suppl. 5):V159-93.

136. Ackerman SE, Gonzalez JC, Gregorio JD, et al. TLR7/8 immune-stimulating antibody conjugates elicit robust myeloid activation leading to enhanced effector function and anti-tumour immunity in pre-clinical models. Cancer Res 2019:79(Suppl. 13):1559.

137. Hinner MJ, Bel Aiba RS, Jaquin TJ, et al. Tumor-localized costimulatory T-cell engagement by the 4-1BB/HER2 costimulatory T-cell engagement by the 4-1BB/MER2 2019;25:5878-89.

138. Piha-Paul S, Bendell J, Tolcher A, et al. O82 A phase 1 dose escalation study of PRS-343, a HER2/4-1BB bispecific molecule, in patients with HER2+ malignancies. I Immunother Cancer. 2020;8. doi: 10.1136/LBA2019.2 [Online ahead of print]. 